\title{
Exploiting a Direct Detection Camera for In-Situ Microscopy
}

\author{
Eric A. Stach, ${ }^{1}$ Dmitri Zakharov, ${ }^{1}$ Rosa Diaz Rivas, ${ }^{1}$ Paolo Longo, ${ }^{2}$ Matthew Lent, ${ }^{2}$ Alexander \\ Gubbens, ${ }^{2}$ and Cory Czarnik ${ }^{2}$ \\ 1 Center for Functional Nanomaterials, Brookhaven National Laboratory, Upton, NY 11733 \\ 2 Gatan Inc., Pleasanton, CA 94588
}

Direct detection cameras have demonstrated improved sensitivity and resolution compared to scintillator-based cameras due to reduction in electron scatter from back-thinning of the silicon and elimination of optical transfer paths $[1,2]$. While the cryo-EM community is leading the worldwide adoption of direct detection cameras, there is a significant opportunity to leverage the high DQE of these systems across much broader applications space. The Gatan K2 camera is a back-thinned, direct detection camera capable of processing 400 frames per second and $1 \mathrm{~K} \times 1 \mathrm{~K}$, which represents an increase of $>10 \mathrm{x}$ in frame rate compared to conventional CCD and CMOS cameras today. Leveraging this sensor design, and incorporating high speed data communication and processing into the system has allowed development of a new camera (dubbed the "K2-IS") that can run up to 1600 frames per second and process and stream the data to storage in real-time. The output is a series of tens of thousands, or hundreds of thousands of individual images, which can then be analyzed individually or played back as a movie.

The first K2-IS system was installed at the Center for Functional Nanomaterials at Brookhaven National Laboratory in January of 2013, and we report initial results from its use. The system was installed onto an FEI Titan 80/300 Environmental TEM, equipped with a CEOS post-specimen aberration corrector. This system is equipped with several heating holders, as well as 'closed cell' holders for both liquid and gas cell experimentation. We will present movies taken at rates as high as 1600 frames per second during in-situ observations of (1) carbon nanotube growth, (2) semiconductor nanowire growth and (3) real time oxidation and reduction of catalytic materials. The presentation will compare and contrast the data quality that can be obtained through use of the K2-IS, versus a standard Orius 832 camera, in terms of both signal-to-noise and frame rate. The remarkable increase in frame rate allows insight into the kinetics of phase transformations and nucleation and growth in these systems. A 'first example' is shown as Figure 1, wherein the sintering of two $\mathrm{Au} / \mathrm{Cu}$ catalyst nanoparticles [3] dispersed onto a carbon film is imaged using a Tecnai F20 operated at $200 \mathrm{kV}$ (at the Gatan Corporation, prior to installation at the CFN), with sequential images acquired with $2.5 \mathrm{~ms}$ exposure time.

Unlike pioneering efforts to work in the 'ultrafast' regime [4,5], the direct detector is compatible with 'conventional' in-situ TEM experimentation - it is possible to observe the dynamic changes in materials structure for extended periods of time, capturing images in a continuous fashion with millisecond time resolution for up to 15 minutes at a time. The amount of data produced is staggering - this 15 minutes leads to the acquisition of 4 terabytes of information, presenting a significant challenge in data transfer and data processing. We will describe how we are utilizing the resources existent at Brookhaven associated with activities in high-energy physics (the ATLAS Collaboration [6]) to transfer, manipulate and analyze the contents of these enormous data streams. We will describe - at a high level - how to deal with these large data sets in terms of transfer within BNL and to users at remote locations, including transatlantic. As identified in a recent DOE Workshop Report [7], the 'data tsunami' produced by advanced detectors can enable fundamentally new modes of discovery. We will present 
our very initial findings in dealing with this data stream, and discuss how this can lead to new opportunities in understanding nanostructure nucleation and growth, as well as the operation of catalysts in their working environments.

1. Turchetta, R., et al., Nucl. Instr. and Meth. A 458 (2001).

2. Contarato, D., et al., Nucl. Instr. and Meth. A 635 (2011).

3. The authors would like to thank Dr.Vittorio Morandi at CNR-IMM Bologna, Italy for providing the $\mathrm{Au} / \mathrm{Cu}$ nanoparticle sample.

4. King, W. E. et al., J. Appl. Phys. 97, 111101 (2005).

5. A.H. Zewail, Science, 328, 187, 2010.

6. http://www.bnl.gov/atlas/computing.php

7. http://science.energy.gov/ /media/ascr/pdf/research/scidac/ASCR_BES_Data_Report.pdf

8. Research carried out at the Center for Functional Nanomaterials, Brookhaven National Laboratory, which is supported by the U.S. Department of Energy, Office of Basic Energy Sciences, under Contract No. DE-AC02-98CH10886
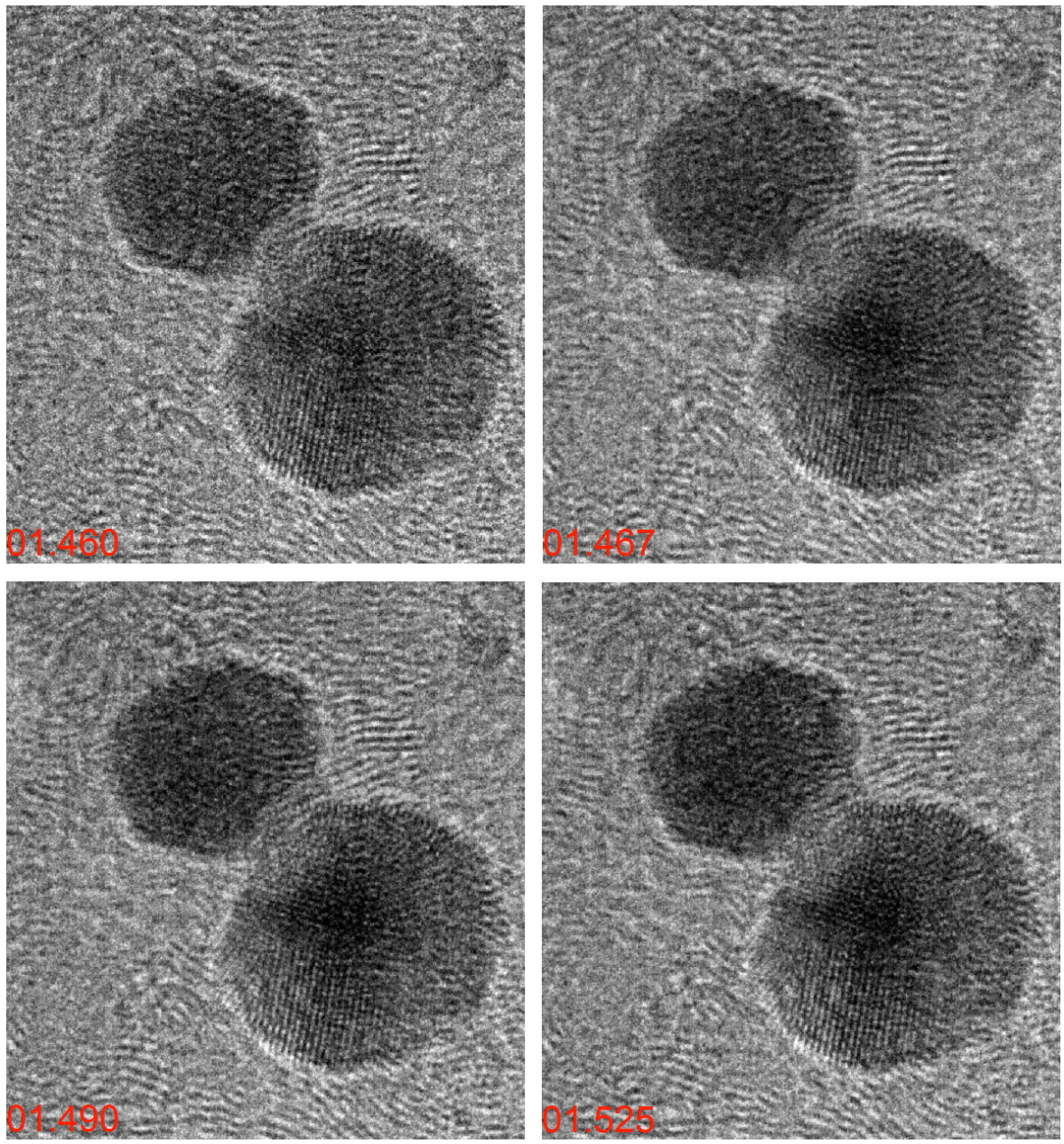

Figure 1: Phase contrast images of the sintering of $\mathrm{Au} / \mathrm{Cu}$ nanoparticles. The images were obtained at a 2.5 millisecond frame rate in a continuous video stream. Individual images that capture the dynamic changes that occur at the interface during sintering are shown. 